\title{
Stevens-Johnson syndrome/toxic epidermal necrolysis spectrum
}

INSERM

\section{Source}

INSERM. (1999). Orphanet: an online rare disease and orphan drug data base. StevensLohnson syndrome/toxic epidermal necrolysis spectrum. ORPHA:95455

Toxic epidermal necrolysis (TEN) is an acute and severe skin disease with clinical and histological features characterized by the destruction and detachment of the skin epithelium and mucous membranes. 